\title{
CONCEPT OF THE HEXA-QUAD BIMORPH WALKING ROBOT AND THE DESIGN OF ITS PROTOTYPE
}

\author{
Dominik WOJTKOWIAK*, Krzysztof TALAŚKA*, Ireneusz MALUJDA*
}

\author{
"Chair of Basics of Machine Design, Faculty of Machines and Transport, \\ Poznan University of Technology, ul. Piotrowo 3, 60-965 Poznań, Poland \\ dominik.wojtkowiak@put.poznan.pl, krzysztof.talaska@put.poznan.pl, ireneusz.malujda@put.poznan.pl
}

received June 2017, revised 16 March 2018, accepted 20 March 2018

\begin{abstract}
Present-day walking robots can increasingly successfully execute locomotive as well as manipulative functions, which leads to their expansion into more and more applications. This article presents the design of a hexa-quad bimorph walking robot with the ability to move at a relatively high speed in difficult terrain. It also has manipulation capabilities both at a standstill and in motion. This feature of the robot is made possible by the ability to easily change the configuration from six-legged to four-legged by elevating the front segment of its body. Presented prototype will be used in further research to develop the hexa-quad bimorph walking robot.
\end{abstract}

Key words: Walking Robot, Hexa-Quad, Bimorph, Hexapod, Quadruped

\section{INTRODUCTION}

There have been significant advances in scientific and technical aspects of walking robots. The changes are noticeable both in design of robot components and motion control. Increasingly advanced designs, varying in size, come to life. To put their legs in motion a broad range of actuators are used - from electric and combustion motors to pneumatic and hydraulic actuators to those using intelligent materials. Modern walking robots can not only move in difficult, rough terrain and different types of surface (ice, sand, etc.), but also climb vertical walls (Saunders et al., 2006) or move under water (Kim and Jun, 2014). Algorithms of motion control and advanced sensors allow generating quick gaits, retaining stability in nearly every condition and even providing movement continuity in case of damaging one of the robot legs (Ignaki, 1998). More and more often walking robots gain motion autonomy thanks to artificial neural networks. This way they acquire machine learning capabilities and artificial intelligence.

Locomotive capabilities of walking robots are well described in scientific literature of the subject. Much less focus is placed on manipulative functions, which walking robots can execute as effectively as locomotive functions. This is what lies behind the concept of the hexa-quad bimorph walking robot whose objective is to combine both functions. The term bimorphism in the context of walking robots means properties that enable two configurations within one design, with no need for changeovers. The concept of the hexa-quad bimorph walking robot came to life following the attempt to design an autonomous walking robot with two key properties:

- the ability to move in difficult terrain with the highest speed possible,

- the ability to manipulate objects both in motion and at a standstill.

Depending on its size, this type of walking robots can have multiple applications, both military (space exploration, scouting and bomb disposal) and civil (mining and forestry).

While analysing the existing, present-day design solutions for walking robots what can be found is partial, but not complete, execution of the functional properties of hexa-quad bimorph walking robots.

Lewinger (2005) presented the design of six-legged walking robot, called BILL-Ant, capable of manipulating objects. In designing the robot, the biological template of the insect, namely the ant, was used. According to the author's assumption, this robot can automatically move on uneven surfaces and manipulate objects in its environment. The robot's advanced locomotive capabilities are owed to six legs with three active degrees of freedom. This type of design makes it possible for the robot foot to reach any point in the working space of the leg. The design element that allows object manipulation is the robot's head, which is equipped with the grabbing device in the form of mandibles. Thanks to three degrees of freedom the robot's head can effectively manipulate the object. The main limitation for this type of design is the unfavourable distribution of weight, which leads to easy destabilization and poor manipulation capabilities while in motion.

The group of walking robots that are successful in executing the locomotive function in difficult terrain while moving at sufficiently high speeds, are designs characterized by a segmented body with active or passive joints. Tang (2015) presented the design of the modular multi-legged walking robot consisting of three or more identical segments. Each segment has one pair of legs with merely one degree of freedom. Between particular segments there is a single axial revolute joint allowing their mutual rotational movement around the vertical axis of the robot. This made it possible to achieve quick gait despite using only a small number of drives. In turn, Bartsch (2012) presented the design of a walking robot with a two-segment body with a controllable joint placed between the segments. The joint allows rotational movement around the axis lateral to the direction of robot move- 
ment. This makes it possible to generate gait algorithms allowing motion through uneven terrain. However, none of these designs executes manipulative functions.

In Kim's work (2014) the design of a six-legged walking robot whose main functional feature was movement under water was presented. The robot was called Little Crabster. Its design is typical for a hexapod with a rigid body, which ensures its gait is quick and statically stable. The robot has front legs executing both locomotive and manipulative functions. To manipulate objects a gripping device extends out of its leg. This type of solution is used to work on objects rather than to move them. It follows from the need to keep the machine's centre of gravity within the support polygon. The problem was partially solved by leaving at least one leg in the support phase, which significantly reduces manipulation capabilities. Placing an extendable gripping device inside the leg requires sufficient space, which means this solution can only be implemented in larger robots.

The next example of a walking robot construction which can fulfill analyzed properties is LAURON V presented by Roennau (2014). Its legs have four independent joints each in order to cope with steep inclines and large obstacles as well as to manipulate objects by using the front legs. It has autonomy of operation, robustness, terrain adaptability and large payload capacity, which makes it highly suitable to for field applications. However, shifting the center of mass can be achieved only by the angular configuration of the legs, which means it will be difficult to control its position during manipulation in motion.

Another interesting construction was presented by Boston Dynamics and was called SpotMini. It is a quadruped with an optional robotic arm ended with a precise gripper which is mounted on the top of the front part of its body. Because its kinematics is biologically inspired it can easily move in rough terrain like a real animal. On the other hand, during the gait generation it is necessary to use dynamic stable gaits instead of static ones in order to achieve full maneuverability.

The last design with traces of bimorphism is the robot MANTIS designed within the LIMES project and presented by Manz (2013). During its operation the robot can assume either of two postures - the locomotive or the manipulative one. This design offers extremely good stability parameters and manipulation capabilities. However, the solution requires high power drives as the rotation of the entire body is necessary. Additionally, the robot design necessitates that object manipulation takes place at a standstill. It also has a negative impact on surmounting obstacles in motion.

Other traces of bimorphism can be also noticed in (Kalouche 2015), where the reconfigurable legged robot with series-elastic actuators was introduced. This type of construction consists of multiple modules that can be easily adapted to quadruped, hexapod or biped configuration. This way, by proper configuration, we can gain the robot locomotion and manipulation abilities.

The objective of this paper is presenting the concept of the hexa-quad bimorph six-legged walking robot, which combines the advantages of the above mentioned designs and eliminates their imperfections. Moreover the goal was to show the construction of the prototype research model, which can be used in further tests in order to develop the idea of a hexa-quad bimorph walking robot by formulating mathematical and physical models. These models can make it possible to describe the design and motion control algorithms as well as the process of designing walking robots capable of executing the above mentioned functions and applications.

\section{THE DESIGN OF THE HEXA-QUAD BIMORPH SIX-LEGGED WALKING ROBOT}

\subsection{The kinematic structure of the hexa-quad bimorph walking robot}

The kinematic structure of the hexa-quad bimorph walking robot is presented in Fig. 1. The construction consists of threesegment body containing the front segment 1 , the middle segment 2 and the rear segment 3 . Between the front segment and the middle segment there is an active double axial joint 7 , which allows the rotation of the front segment relative to the vertical axis $Z$ and the lateral axis $X$. The rear segment is connected with the middle segment with an active single axial joint, which allows its rotation around the vertical axis $Z$. Each body segment is assigned only one pair of limbs. The locomotive legs, whose only function is executing robot motion in space, are attached to the middle and rear segments. Front legs, apart from basic locomotive functions, are also used to manipulate objects. They are - as a result - manipulative and locomotive legs. Additionally, the hexaquad bimorph six-legged walking robot is equipped with an extendable weight system 8 built into the rear body segment. Its purpose is making regulation of the robot's centre of gravity possible (Wojtkowiak, 2017).

Bimorphism of this type of design has to do with its ability to move easily from hexapod configuration to quadruped configuration or the other way around. Both robot configurations are presented in Fig. 2 and Fig. 3.

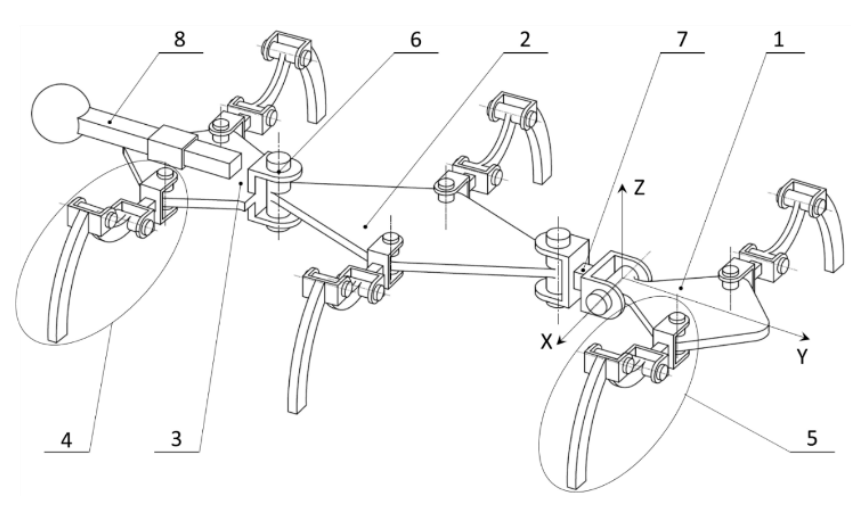

Fig. 1. Design schematic of the hexa-quad bimorph six-legged walking robot: 1 - front body segment, 2 - middle body segment, 3 - rear body segment, 4 - locomotion leg, 5 - locomotion and manipulation leg, 6 - single axial joint, 7 - double axial joint, 8 - movable weight system; $X$ - lateral axis, $y$ - longitudinal axis, Z - vertical axis

The assumption is that in this configuration the robot should achieve the highest possible speed while moving through uneven terrain. The number of legs in the basic configuration, that is the one where all the robot legs are used to generate gait, is predicated mainly on the need for the static stability of gait in both configurations. To meet this criterion, the robot needs to have at least six legs, which means that in the alternative configuration it will move on four legs. Choosing a larger number of legs would allow higher speeds of statically stable gaits but it would significantly reduce the weight and size of the robot and greatly complicate both the design and motion control algorithms (Todd, 1985). 


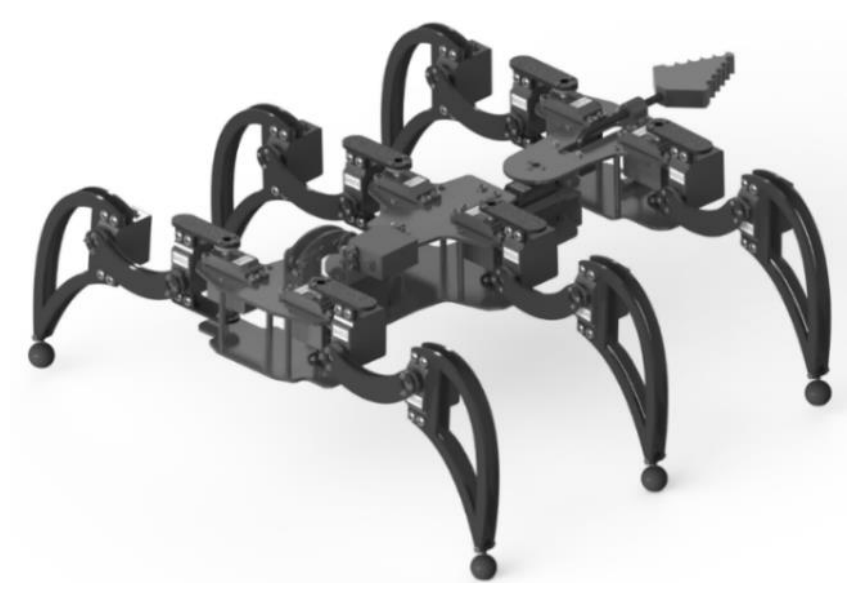

Fig. 2. 3D model of the hexa-quad bimorph walking robot in HEXAPOD configuration

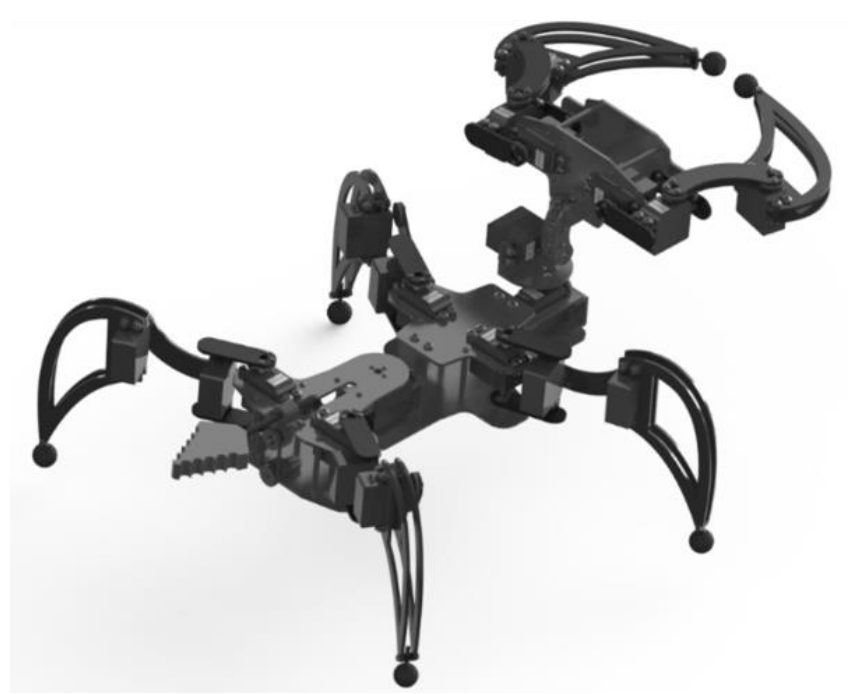

Fig. 3. 3D model of the hexa-quad bimorph walking robot in QUADRUPED configuration

While designing the construction the choice was to minimize the number of legs so as not to complicate an already complex kinematic structure. Because the body is made of three segments, with active joints between the segments, it is possible to support the machine movement by adequately steering the robot body. Thanks to this, with a smaller number of legs, faster, statically stable gaits are achieved. Adequately using the drives of the body joints it is also possible to surmount obstacles in the form of naturally-occurring terrain or man-made obstacles, e.g. street kerbs.

Transformation into the alternative QUADRUPED robot is executed by elevating the front body by means of the drive in the double axial joint. In this configuration mainly the second key function of the hexa-quad bimorph walking robot is executed - manipulating objects while in motion or at a standstill. The robot can perform the object gripping and moving operations as well as perform operations on the objects, e.g. pressing buttons or pushing. To execute the above mentioned functions locomotive and manipulative legs are used. Moving around on the four remaining legs, the robot is forced to use the only statically stable gait - crawl. As a result, to achieve higher motion speeds in the alternative configuration, it is necessary to use dynamically stable gaits.

\subsection{Physical research model}

Based on the above described kinematic structure the design of the research model was created. Its $3 D$ model is presented in Fig. 2. The basic function of the designed machine is performing specific analyses and designing motion control algorithms for hexa-quad bimorph walking robots. Due to a testing nature of the design, the machine was designed in a way that ensures simplicity of manufacturing and assembly. This construction is a prototype whose testing will contribute to building walking machines adapted to specific applications, both military (e.g. minesweeping robot) and civilian (e.g. mining machines).

The most important component of every walking robot are pedipulators - that is the legs of the robot executing basic locomotive functions. Their design has an influence on nearly all the features related to stability and generation of robot gait. In the presented design the biological structure of the insect was used. However, the ratio of lengths is different than in the standard insect structure being 1:2,3:4 instead of 1:4:5. Each limb consists of three links, hip segment $35 \mathrm{~mm}$ long, thigh segment $80 \mathrm{~mm}$ long and shank segment $140 \mathrm{~mm}$ long. That size of the leg makes it possible to achieve the step length $66 \mathrm{~mm}$ and the velocity of movement $33 \mathrm{~mm} / \mathrm{s}$. Kinematic analysis of this type of pedipulator was performed and presented in previous papers (Wojtkowiak, 2016). The foot structure of the insect is much more energyefficient than the foot structure of reptiles or mammals. The features inspired by this biological structure are a three-segment body with one pair of legs assigned to each segment, and multifunctionality of the feet (Zielińska, 2014 and Morecki et al., 1999).

Pedipulator of the hexa-quad bimorph walking robot (Fig. 4) consists of three links. The first link consisting of two identical elements $1 \mathrm{a}$ and $1 \mathrm{~b}$ is called the hip segment. It is connected with the body by the joint allowing rotation around the vertical axis. The middle link 2, called the thigh segment, and the final link 3, the socalled shank segment, have the ability to rotate around the longitudinal axis. At the end of this link the robot's foot 4 is located. Its main functions are to provide adequate friction between the robot and the surface, necessary to move around, to absorb foot strike and to enable the assembly of pressure sensors needed for testing static stability. The three-segment construction of the walking robot leg ensures that the foot can achieve any position within the working area of the pedipulator. All the segments are made from flat cranks of curvaceous shapes, connected with each other by means of servomotors 5, 6 and 7, which control the angular position of particular links, generating the robot's gait. In order to avoid complicating the design of the research model, the only difference between manipulation and locomotion legs and other pedipulators is the additional reinforcement in the middle segment in the form of the second flat crank to increase the stiffness of the leg. At the end of the shank segment there is a foot embedded on a ball pivot with a spring element 8 . To ensure a sufficiently stiff connection between the leg and the body, the upper part 1a of the hip segment is connected to the servomechanism and the lower part $1 \mathrm{~b}$ can rotate thanks to the axis 9 and the bearing set 10 in the lower plate of the body 11. The shape of each segment was designed based on the FEM analyses together with kinematic analyses, which helped to describe the operation zone of the pedipulator and prevent dangerous collisions during work (Wojtkowiak, 2016). Additionally, authors tried to reduce the weight of the limbs, which decreased the inertia and needed torque. 


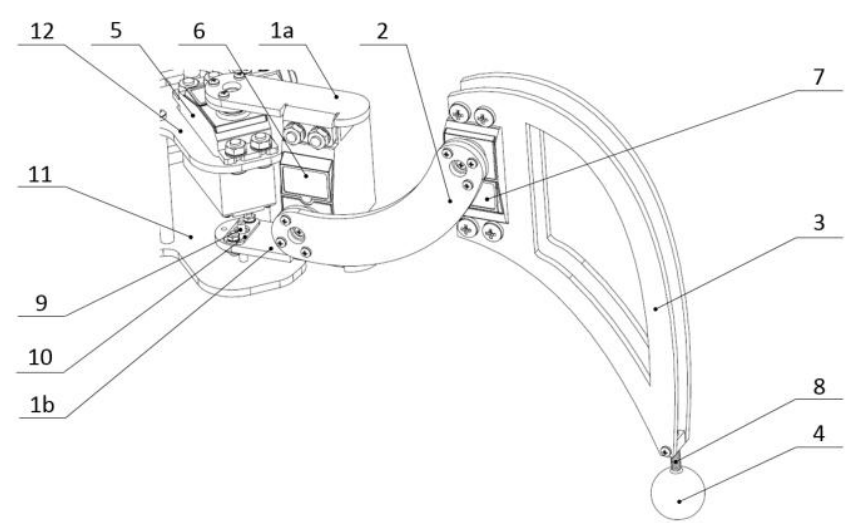

Fig. 4. Pedipulator structure of the hexa-quad bimorph walking robot: $1 \mathrm{a}$ - upper part of the hip segment, $1 \mathrm{~b}$ - lower part of the hip segment, 2 - thigh segment, 3 - shank segment, 4 - foot, 5 - hip segment servomotor, 6 - thigh segment servomotor, 7 - shank segment servomotor, 8 - foot pivot, 9 - axis, 10 - bearing set, 11 - lower plate of the body, 12 - upper plate of the body

Characteristic elements of the hexa-quad bimorph walking robot design are the joints between body segments and the extendable weight system. Single axial joint (Fig. 5), between the middle and the rear body segment consists of the servomotor 1 responsible for rotation around the vertical axis. The motor is based on two C-channels mounted on the lower plate of the middle body 3 . The upper plate of the rear body 4 is adequately profiled and mounted directly to the servomotor. In the double axial joint (Fig. 6) that can be found between the front and the middle body segment, rotation around the vertical axis is executed in an almost identical way, the only difference being that the turning plate 4 is mounted on the servomotor 1 . The servomotor 5 responsible for rotation around the lateral axis is screwed onto the turning plate. To the servo motor shaft is attached the elevating crank whose tip is connected by means of the $\mathrm{C}$-channel 7 with the upper plate of the front body segment 8.

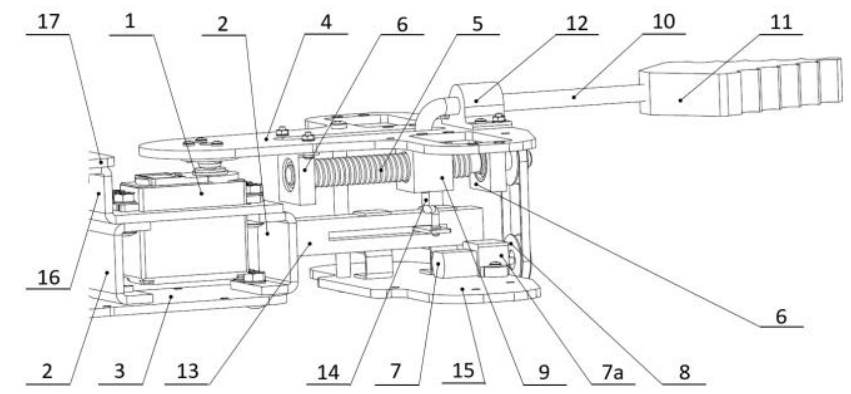

Fig. 5. Structure of the single axial joint and the extendable weight system: 1 - vertical servo motor, 2 - supporting C-channel, 3 - lower plate of the middle body segment, 4 - upper plate of the rear body segment, 5 - trapezoidal thread screw, 6 - bearing support, $7-\mathrm{DC}$ motor, $7 \mathrm{a}$ - motor mount, 8 - transmission belt, 9 - nut, 10 - slider 11 - counterweight, 12 - linear sliding bearing, 13 - sliding potentiometer, 14 - connector, 15 - the lower plate of the rear body segment, 16 - handle of the body servomotor, 17 - the upper plate of the middle body segment

Due to limited enclosure space, the design of the extendable weight system is based on the screw/nut drive mechanism. The system is presented as a schematic in Fig. 7 and its structure is presented in Fig. 5. The screw 5 with the symmetrical trapezoidal thread with support bearings 6 at its tops is driven by the DC motor 7 by means of the belt transmission 8 . As a result of the rotary motion of the screw the translational motion of the nut starts. The slider 10 embedded in the linear bearing 12 is rigidly attached to the nut. At the end of the arm is the counterweight 11. This kind of solution excludes the possibility of subjecting the screw to the bending moment. In order to read the current position of the nut it is connected to the sliding potentiometer 13.

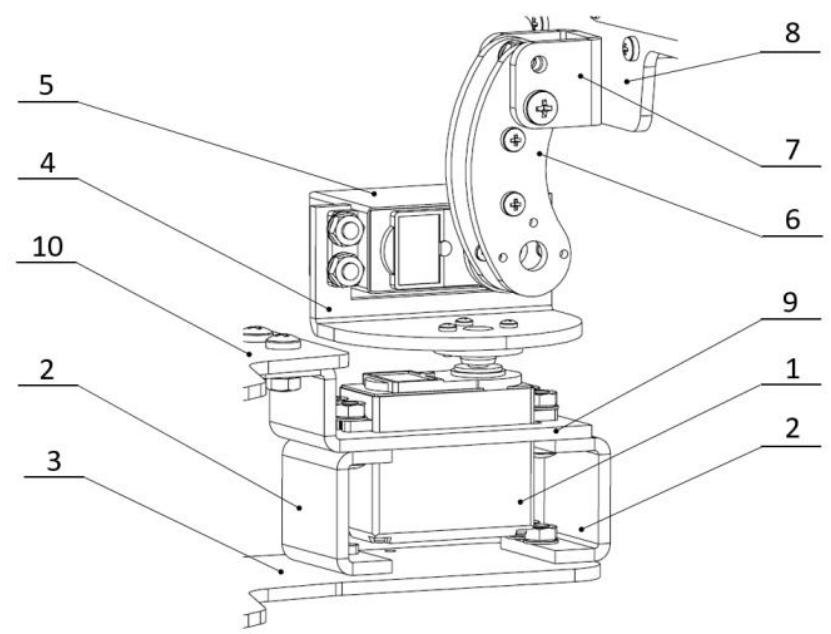

Fig. 6. Structure of the double axial joint of the hexa-quad bimorph walking robot: 1 - vertical servomotor, 2 - supporting C-channel, 3 - lower plate of the middle body segment, 4 - turning plate, 5 - horizontal servomotor, 6 - elevating crank, 7 - angular plate for mounting the elevating crank, 8 - the upper plate of the front body segment, 9 - handle of the body servo motor, 10 - the upper plate of the middle body segment

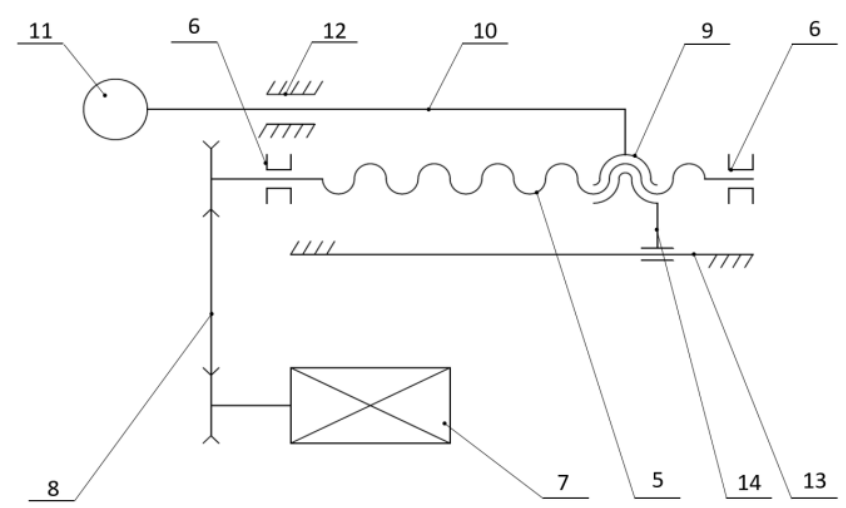

Fig. 7. Kinetic schematic of the extendable weight system: 5 - screw with trapezoidal thread, 6 - bearing support, 7 - DC motor, 8 - belt transmission, 9 - nut, 10 - arm, 11 - counterweight, 12 - longitudinal bearing, 13 - sliding potentiometer, 14 - connector

Apart from the mechanical construction a very important stage in designing walking robots is the choice of actuators, sensors, drivers and power supply systems. 3D model of the testing prototype with necessary electronics is presented in Fig. 8.

In order to select the drives, the analysis of maximum load moments of particular joints of the robot needs to be performed. The following analyses were performed and presented in the previous work (Wojtkowiak, 2017). The presented research model is driven by 21 analogue servomotors with range of motion be- 
tween $0^{\circ}$ and $180^{\circ}$. Despite small size and weight, they have a relatively big moment and sufficient speed with the $6 \mathrm{~V}$ power supply. An additional advantage of choosing these motors is the fact that their components - gears and the serrated output shaft are made from metal, not plastics. Using metal connectors ensures required stiffness of the serrated connection between the output shaft and the connector. The extendable weight system is driven by the $D C$ motor with the rotational speed of $70 \mathrm{rpm}$ and supply voltage of $6 \mathrm{~V}$.

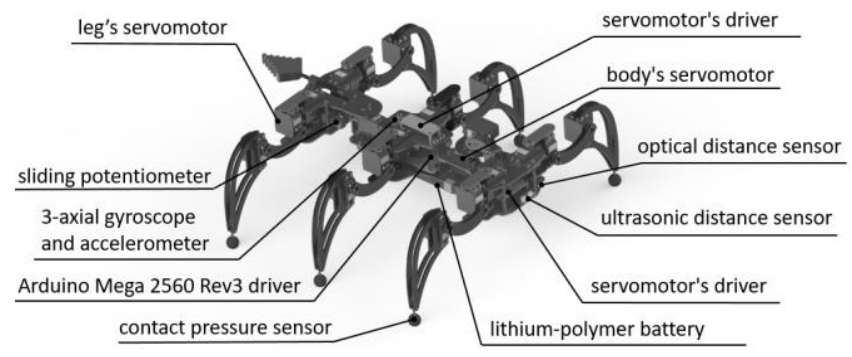

Fig. 8. 3D model of the hexa-quad bimorph walking robot with sensors and drivers

In order to obtain a certain degree of operational autonomy of the tested prototype, it is equipped with a number of sensors. Because of the application of the testing prototype, which is to enable research in order to describe the kinematics and the steering method, the robot was equipped only with the basic sensors. In the front and in the rear of the robot there are two ultrasound distance sensors with a range of $2-400 \mathrm{~cm}$, whose role is to detect obstacles on the robot's path. On the lower plate of the front body segment, from below, the optical distance sensor with a range of $4-30 \mathrm{~cm}$ is mounted. In the basic configuration its role is to monitor clearance between the front body segment and the surface providing information about the characteristics of the terrain where the robot is moving. In the alternative configuration its role is to read the position of the objects that are being manipulated. A very important piece of equipment, as far as testing dynamic stability and the influence of terrain slope on the robot's gait, is the three axial accelerometer and gyroscope mounted on the middle body segment. In order to test static stability on the basis of foot pressure, each leg is equipped with the resistance pressure sensor with the measurement range between $0.2 \mathrm{~N}$ and $20 \mathrm{~N}$. So as to measure power consumption of the servomechanism the robot is equipped with the power sensor.

In order to control selected actuators and analyze signals from the above described sensors the robot is equipped with Arduino Mega 2560 Rev 3 driver based on ATmega2560 microcontroller. The system contains 54 input/output pins, of which 15 can be used as PWM outputs, 16 analog inputs and 4 UART hardware serial ports, an SPI and I2C. The system is powered with 7-12 V input voltage. In order to control servomotors two 16-channel servomotor controllers Adafruit containing accurate 12-bit PWM signal generator are used. In turn, the DC motor is controlled by the system based on the $\mathrm{H}$ bridge allowing the change in rotation direction and speed regulation. In order to separate control systems from servomotors that introduce interferences a separate power supply is used. The testing prototype can be powered from the lithium polymer battery located at the bottom of the front body segment or from separate lab power supplies. The battery that supplies power to the electronic components has the capacity of $1300 \mathrm{mAh}$ whereas the battery that supplies power servomotors has the capacity of $3300 \mathrm{mAh}$. Both batteries are two cell and have nominal voltage of $7.4 \mathrm{~V}$ and big current efficiency (30C). Although this capacity will be enough only for maximum 30 minutes of operation.

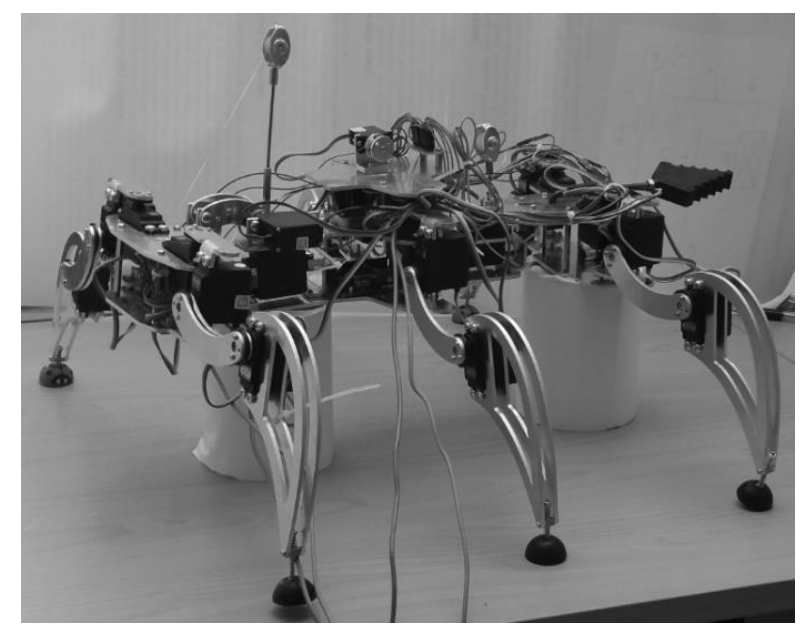

Fig. 9. Hexa-quad bimorph walking robot prototype

On the basis of the formulated design the testing prototype presented in Fig. 9 was built. Its overall dimensions do not exceed $500 \mathrm{~mm}$ in length and $300 \mathrm{~mm}$ in width. The height of the bimorph hexa-quad walking robot is less than $200 \mathrm{~mm}$ in hexapod configuration, measuring from the surface on which it stands to the top plate of the base (without accessories mounted on its back) and rise to $350 \mathrm{~mm}$ after changing its configuration to quadruped. Total weight of the robot is approximately $3 \mathrm{~kg}$. The payload of the machine should not exceed $0.5 \mathrm{~kg}$, but could be increased using heavier retractable weight.

\section{GAIT STABILITY}

Gait stability is one of the most important parameters influencing a number of functional features related to walking robots, from mechanical construction to control algorithms and generating steps. There are two types of stability in walking robots: static stability and dynamic stability. The measurement value to determine stability margin can be distance, angle, energy, moment or force. Many stability criteria have been formulated on their basis. With appropriate assumptions, the same methods can be used to determine both types of stability. One example is the energy stability margin, which involves measuring the difference between the initial potential energy and the actual potential energy. If we consider that all external forces including the force of gravity act on the centre of gravity of the robot we get the dynamic energy stability margin (Hajiabadi, 2013; Hirsoe, 2001).

In the above mentioned research projects attention was focused solely on static stability. The main assumption of static stability of walking robots is retaining centre of gravity or its projection inside the support polygon. This region is defined as a support polygon, a two-dimensional figure created from coordinates of tips of all supporting legs projected onto a support plane. These coordinates can be calculated based on the kinematic model of the leg (Wojtkowiak, 2016). In order to determine which legs are currently in a support phase, pressure sensors can be used. As a quantitative criterion static stability margin (SSM) and 
longitudinal static stability margin (LSM) were used - Fig. 10. Longitudinal static stability margin is defined as the shorter one out of the distances between the projection of centre of gravity on the support plane and the edge of the support polygon as measured along the direction of the walking robot's speed vector. In turn, static stability margin is defined as the shortest distance between the aforementioned projection and the edge of the support polygon. (Inagaki, 1998; Hung, 2005). These methods can be used in cases of negligible inertia and absence of forces counteracting the robot's movement. However, irregularities of the surface where the robot is moving around do not have influence on the adequacy of the results obtained by means of one of the above mentioned methods (Garcia, 2002).

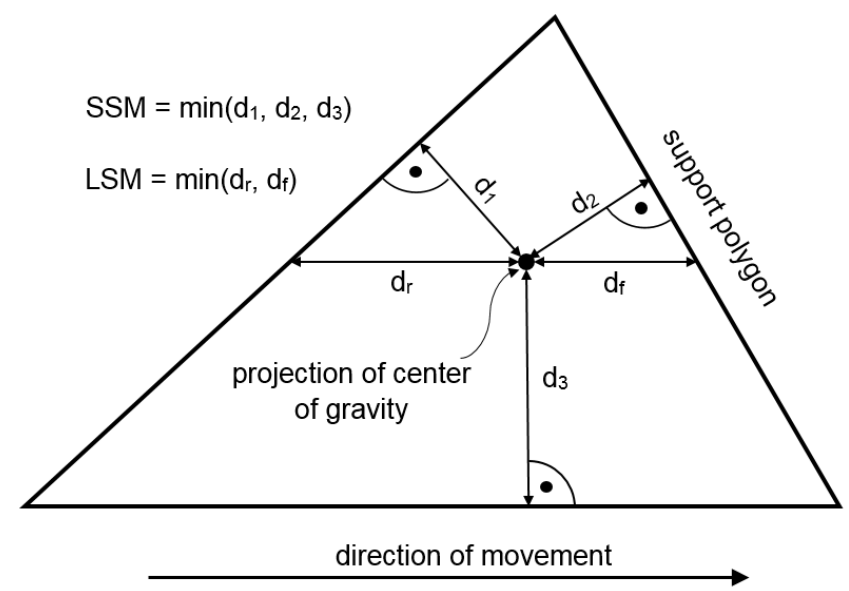

Fig. 10. Support polygon together with indicated stability margins

\section{SUMMARY}

In this article the design of the hexa-quad bimorph walking robot was presented. The key advantage of this robot is, that it construction provides an ability to move easily from hexapod configuration to quadruped configuration or the other way around. This means, it can combine advantages of both four-legged and sixlegged robots. Basically its main function is to achieve the highest possible speed while moving through uneven terrain. Additionally, robot can manipulate objects while being in motion or at a standstill. In order to gain desired parameters of the hexa-quad bimorph walking robot, presented prototype must be tested by making a series of researches, which contain stability and control algorithms tests.

\section{REFERENCES}

1. Bartsch S. (2012), Development, control, and empirical evaluation of the six-legged robot SpaceClimber designed for extraterrestrial crater exploration, dissertation, Bremen, University of Bremen.

2. Boston Dynamics website https://www.bostondynamics.com/robots [Access date: 26.10.2017].

3. Garcia E., Estremera J., Gonzalez-de-Santos P. (2002) A comparative study of stability margins for walking machines. Robotica, 20, 595-606.

4. Garcia E., Estremera J., Gonzalez-de-Santos P. (2002), A classification of stability margins for walking robots, Proceedings of CLAWAR, Paris, France.
5. Hajiabadi M.M.A. (2013), Analytical workspace, kinematics, and foot force based stability of hexapod walking robots, dissertation, Worcester: Worcester Polytechnic Institute.

6. Hirsoe S., Tsukagoshi H., Yoneda K. (2001), Normalized energy stability margin and its contour of walking vehicles on rough terrain, International Conference on Robotics \& Automation, Seoul Korea.

7. Hung M-H., Cheng F-T., Lee H-L. (2005), Orin DE. Increasing the stability margin of multilegged vehicles through body sway. J Chin. Inst. Eng, 28, 39-54.

8. Inagaki K. (1998), A gait study for one-leg-disabled hexapod robot, Advanced Robotics, 12, 593-604.

9. Kim J-Y., Jun B-H. (2014), Design of six-legged walking robot, Little Crabster for underwater walking and operation, Advanced Robotics, 28, 77-89.

10. Kolouche S., Rollinson D., Choset H. (2015), Modularity for maximum mobility and manipulation: control of a reconfigurable legged robot with series-elastic actuators, Proceedings of the IEEE International Symposium on Safety, Security and Robotics (SSRR), 1-8.

11. Lewinger W.A, Branicky M.S., Quinn R.D. (2005), Insect-inspired, actively compliant hexapod capable of object manipulation, Proceedings of CLAWAR, Londom, 65-72.

12. Manz M., Bartsch S., Kirchner F. (2013), MANTIS - a robot with advanced locomotion and manipulation abilities, Proceedings of Symposium on Advanced Space Technologies in Robotics and Automation, Noordwijk the Netherlands.

13. Morecki A., Knapczyk.J. (1999), Basics of Robotics - theory and elements of manipulators and robots (in polish), Warszawa.

14. Roennau A., Heppner G., Nowicki M., Dillmann R. (2014), LAURON V: A versatile six-legged walking robot with Advanced Maneuverability, IEEE/ASME International Conference on Advanced Intelligent Mechatronics (AIM), Besançon, France, 82-87.

15. Saunders A., Goldman D.I., Full R.J., Buehler M. (2006), The RiSE climbing robot: body and leg design, Proceedings of The International Society of Optical Engineering, Orlando USA, 6230, 623017.

16. Tang Y., Ma S., Sun Y., Ge D. (2015), Planar legged walking of passive-spine hexapod robot, Advanced Robotics, 29, 1510-1525.

17. Todd D.J. (1985), Walking machines - An introduction to legged robots, Springer, London.

18. Wojtkowiak D., Malujda I., Talaśka K., Magdziak Ł., Wieczorek B. (2017), Influence of the Body Weight Distribution on the Walking Robot's Gait Stability, Proceedia Engineering, 177, 419-424.

19. Wojtkowiak D., Talaśka K., Malujda I. (2016), Computer analysis of insect-like robot leg structure - part 1 - Static Finite-Element analysis, Journal of Mechanical and Transport Engineering, 68(3), 53-62.

20. Wojtkowiak D., Talaśka K., Malujda I. (2016), Computer analysis of insect-like robot leg structure - part 2 - kinematic and dynamic analyses, Journal of Mechanical and Transport Engineering, 68(3), 63-75.

21. Wojtkowiak D., Talaśka K., Malujda I. (2017), The selection of the bimorph walking robot drives based on the dynamic model of its legs (in polish), Inżynieria wytwarzania, Wyd. uczelniane Państwowej Wyższej Szkoły Zawodowej w Kaliszu, in press.

22. Zielińska T. (2014), Walking robots - basics, design, steering and biological patterns, PWN, Warszawa. 